Шляхом математичного моделювання підтверджено високу експериментальну активність $\mathrm{MgAl}_{2} \mathrm{O}_{4} / \mathrm{NiO} / \mathrm{Cr}_{2} \mathrm{O}_{3}$ каталізатора в реакиії глибокого окислення метану. Досліджено поверхневу концентрацію метану та температуру розігріву каталізатора. Визначені значення максимальної температури розіррівання каталізатора - $1085 \mathrm{~K}$, коефіцієнти масо-тепловіддачі. Ефективна робоча поверхня каталізатора - $7640 \mathrm{м}^{2} / \mathrm{м}^{3}$. Дані дослідження можна використовувати в інженерних розрахунках інфрачервоних каталітичних пальників

Ключові слова: окислення метану, питома поверхня, метод Рунге-Кутти, волокневий каталізатор

Путем математического моделирования подтверждена высокая әкспериментальная активность $\mathrm{MgAl}_{2} \mathrm{O}_{\sqrt{ }} \mathrm{NiO} / \mathrm{Cr}_{2} \mathrm{O}_{3}$ катализатора в реакции глубокого окисления метана. Определена концентрация метана на поверхности катализатора. Максимальная температура разогрева катализатора - 1085 К. Рассиитаны коэффициенты массо-теплоотдачи. Эффективная рабочая поверхность катализатора - $7640 \mathrm{~m}^{2} / \mathrm{s}^{3}$. Даннье исследования можно использовать в инженерных расчетах инфракрасных каталитических горелок

Ключевые слова: окисление метана, удельная поверхность, метод Рунге-Кутты, волокнистый катализатор

$$
\text { 口- }
$$

\section{DEVELOPMENT OF MATHEMATICAL MODEL OF METHANE OXIDATION ON FIBROUS CATALYST}

A. Popovich Engineer

Department of General physics and technical mechanics* E-mail: deseretwind@gmail.com

H. Soloviev

$\mathrm{PhD}$, Associate Professor Department of Ecology*

E-mail: solgenn46@gmail.com

V. O r l y k

PhD, Leading Researcher

Gas Institute of the NAS of Ukraine Degtyarivska str., 39, Kyiv, Ukraine, 03113

E-mail: orlykvol@gmail.com

A. Suvorin

Doctor of Technical Sciences, Professor

Department of chemical engineering and ecology

Volodymyr Dahl East Ukrainian National University Tsentralnyi ave., 59-a, Severodonetsk, Ukraine, 93400

E-mail: avsuvorin@rambler.ru

*Institute of Chemical Technologies

Volodymyr Dahl East Ukrainian National University

Volodymyrska str., 31, Rubizhne, Ukraine, 93009

2. Literature review and problem statement

At the moment, specific mathematical models have been developed that allow to determine the optimum characteristics of the reaction equipment, catalysts, and used components. The advantages of mathematical modeling are presented in a number of works. In [1], the authors, using mathematical modeling of the catalytic heater, determine the optimum characteristics of the reactor and the catalyst, providing a specific heat output of $19.35 \mathrm{~kW} / \mathrm{m}^{3}$.

The feasibility of modeling is confirmed by the authors in [2]. Due to the developed model, the dynamics of methane oxidation on nickel is studied, the occurrence of self-oscillation reaction under isothermal conditions due to periodic oxidation-nickel reduction is confirmed. As a result of the study, detailed information is obtained on the mechanism of the reaction, both in stationary and in oscillation regimes.

With the help of analytical calculations, the authors of [3] select an equation that describes well the experimental data of deep oxidation of methane on 1C 12-72 catalyst and improve the flow-circulation method of studying kinetics.

With the help of developed mathematical model, the author [4] study in detail the kinetic mechanism of meth- 
ane oxidation, which includes 213 elementary reactions. The advantage of this model is the high convergence of the experimental and predicted results of methane, oxygen consumption and the formation of carbon monoxide and water. However, the predicted amount of formed nitric oxide is less than the experimental one. What is a significant drawback, since nitrogen oxide is an air pollutant.

In $[5,6]$, the authors use the mathematical model of multi-substrate kinetics, which describes the biocatalytic oxidation of methane. The study of this issue can solve a number of environmental problems associated with the reduction of methane emissions from landfills of solid domestic waste, farms, degassing installations of coal mines.

To improve the process of soft steam reforming, the authors of [7] propose to use the model developed by them. To solve the direct kinetic problem, a method is used based on the third-order Rosenbrock single-iteration formula. In the process of modeling, a solution of a system of differential equations, rigid in terms of components, is found. This makes it possible to determine changes in the concentrations of the reactants from the length of the reactor at a particular temperature or from an array of temperatures.

The authors of [8] present a mathematical model for gas synthesis in a reactor for the filtration combustion of gases. The combustion process is based on a rich methane-air mixture as the basis of the model. As a result of modeling, the composition of the product, the temperature in the combustion zone, and the propagation velocity of the filtration combustion wave are numerically studied. The results of the modeling are confirmed by experimental data.

A mathematical model of the hydrocarbon fuel conversion process inside a thermochemical reactor as an element of thermal protection of a hypersonic aircraft is described in [9]. As a result of the study it is found that the maximum yield of hydrogen is achieved with methane consumption greater than the flow of water.

In the process of modeling the catalytic oxidation of methane on $\mathrm{Ni} / \gamma-\mathrm{Al}_{2} \mathrm{O}_{3}$, the author [10] found that the main oxidant is $\mathrm{OH}^{*}$, and not $\mathrm{O}^{*}$, which is formed as a result of the oxidation of hydrogen on the surface of the catalyst. It has also been found that the growth of the reaction temperature has a positive effect on the dissociation rate of methane and carbon dioxide, but the formation of carbon increases, which leads to a deactivation of the catalyst.

In [11], the authors use modeling to study the features of the course of catalytic steam reforming of methane in a fixed-bed reactor and in a membrane reactor. Due to the developed model, the temperature limits for the maximum conversion of methane are established. Thus, for the first type of reactor, the methane conversion rate of $97.21 \%$ is reached at a temperature of $1250 \mathrm{~K}$, and in the second type reactor the methane conversion rate of $99.79 \%$ is already observed at a temperature of $923 \mathrm{~K}$. It is also found that the hydrogen yield in the reactor of the second type prevails over the first.

The authors of [12] use modeling to optimize the oxidation of methanol on an iron-molybdenum catalyst. The model takes into account the influence of the initial temperature, coolant temperature and the air-to-methanol molar ratio.

In the study [13], a complex kinetic model of methane oxidation on a $\mathrm{Li} / \mathrm{MgO}$ catalyst is developed. The model takes into account the catalytic and gas-phase reaction stages. Using the Langmuir-Hinshelwood-Hougen-Watson velocity equation, the model takes into account the inhibitory effect of carbon dioxide and oxygen on the oxidation reaction. Comparison of the predicted data with the experimental data confirmed the correctness of the calculations.

\section{The aim and objectives of research}

The aim of research is determination of the areas of heterogeneous catalytic oxidation of methane flowing in a laboratory flow reactor and the effective working surface of the catalyst. This will make it possible to derive a microkinetic equation for the rate of methane oxidation and to perform engineering calculations of catalytic burners.

To achieve this aim, it is necessary to solve the following tasks:

- to determine the hydrodynamic performance of the process in a laboratory flow reactor;

- using the criterial equations to determine the coefficients of mass transfer and heat transfer;

- to determine the temperature of the surface heating of the catalyst and the concentration of reagents on the catalyst surface.

\section{Materials and methods of research}

\section{1. The investigated materials and equipment that in}

\section{the experiment}

The experimental data obtained in a flow laboratory reactor in the study of the kinetics of heterogeneous catalytic oxidation of methane on a fibrous catalyst are used as a basis in this study. The procedure for carrying out the experiment to study the kinetics of methane oxidation on a catalyst is similar to the procedure described in [14]. Catalyst: carrier synthetic fibrous material Alxiflex KT 1620 - 51,73\%; adhesive $\mathrm{Si}+\mathrm{SiO}_{2}-18.97 \%$; organic glue $-1,72 \%, \mathrm{MgAl}_{2} \mathrm{O}_{4}-$ $22,07 \% ; \mathrm{NiO}-2.02 \% ; \mathrm{Cr}_{2} \mathrm{O}_{3}-3.49 \%$. For a detailed study of the oxidation mechanism, a complete two-phase mathematical model is used.

The model takes into account:

- kinetic mechanism of methane oxidation in the presence of a catalyst;

- composition of the original components;

- thermal effects of the reaction, which take place in the core of the gas flow and on the catalyst surface;

- mass-heat transfer coefficients, coefficient of thermal conductivity of the catalyst skeleton.

\section{2. Method for determination of the reaction areas} and the working surface of the catalyst

To optimize the calculations for determining the reaction areas, the working surface of the catalyst and the optimum height of the catalyst bed, a complete two-phase mathematical model is developed. As a basis for modeling, a model of microchannels is adopted. This model is characterized by the equality of the total area of the cross-sections of the microchannels and the area of the free section of the catalyst, and the total surface area of heat and mass transfer is equal to the specific surface area of the catalyst. Values of kinematic viscosity, density and other thermophysical parameters are calculated in accordance with the initial composition of the mixture and the bulk load. 
To facilitate calculations, the model assumes:

1. For the gas flow, the ideal displacement model is adopted.

2. There is no gradient in the gas flow and in the catalyst along the radius of the cross section, i. e., the loss of heat through the reactor wall does not lead to the formation of a temperature gradient.

3. All reactions are irreversible.

4. The amount of substance that has reacted for each of the reactions is proportional to the volume of the catalyst (or free volume), and the mass- and heat transfer intensity between the core of the gas stream and the surface depends on the degree of development of the interfacial surface.

5. The distribution of the gas parameters along the length of the reactor corresponds, in view of the low inertia of the processes in the gas phase, the stable state with the current temperature distribution of the catalyst.

When performing the calculations, the composition of the reaction mixture and the reactions that take place both on the catalyst and in the free volume are taken into account, the data are presented in Table 1. The composition of the gases includes nitrogen $-\mathrm{N}_{2}$, oxygen $-\mathrm{O}_{2}$, water vapor $-\mathrm{H}_{2} \mathrm{O}$, carbon monoxide and carbon dioxide - $\mathrm{CO}$ and $\mathrm{CO}_{2}$, monoxide and nitrogen dioxide - NO and $\mathrm{NO}_{2}$, as fuel: methane $-\mathrm{CH}_{4}$, hydrocarbons $-\mathrm{C}_{\mathrm{n}} \mathrm{H}_{\mathrm{m}}$ and hydrogen $\mathrm{H}_{2}$.

Table 1

Reactions that describe catalytically-stabilized oxidation of methane on the catalyst surface and in a gas stream

\begin{tabular}{|c|c|}
\hline On the catalyst surface & In the gas flow \\
\hline $\begin{array}{c}\mathrm{CH}_{4}+2 \mathrm{O}_{2}=\mathrm{CO}_{2}+2 \mathrm{H}_{2} \mathrm{O} \\
-\mathrm{Q}_{1}\left(-453 \cdot 10^{3} \mathrm{~kJ} / \mathrm{kmol}\right)\end{array}$ & $\begin{array}{c}\mathrm{CH}_{4}+2 \mathrm{O}_{2}=\mathrm{CO}_{2}+2 \mathrm{H}_{2} \mathrm{O} \\
-\mathrm{Q}_{15}\left(-453 \cdot 10^{3} \mathrm{~kJ} / \mathrm{kmol}\right)\end{array}$ \\
\hline $\begin{array}{l}\mathrm{CH}_{4}+\mathrm{O}_{2}=\mathrm{CO}+\mathrm{H}_{2}+\mathrm{H}_{2} \mathrm{O} \\
-\mathrm{Q}_{2}\left(-283 \cdot 10^{3} \mathrm{~kJ} / \mathrm{kmol}\right)\end{array}$ & $\begin{array}{c}\mathrm{CH}_{4}+\mathrm{O}_{2}=\mathrm{CO}+\mathrm{H}_{2}+\mathrm{H}_{2} \mathrm{O} \\
-\mathrm{Q}_{16}\left(-283 \cdot 10^{3} \mathrm{~kJ} / \mathrm{kmol}\right) \\
\end{array}$ \\
\hline $\begin{array}{c}\mathrm{CH}_{4}+\mathrm{H}_{2} \mathrm{O}=\mathrm{CO}+3 \mathrm{H}_{2} \\
+\mathrm{Q}_{3}\left(+204,3 \cdot 10^{3} \mathrm{~kJ} / \mathrm{kmol}\right) \\
\end{array}$ & $\begin{array}{c}\mathrm{CH}_{4}+\mathrm{H}_{2} \mathrm{O}=\mathrm{CO}+3 \mathrm{H}_{2} \\
+\mathrm{Q}_{17}\left(+204,3 \cdot 10^{3} \mathrm{~kJ} / \mathrm{kmol}\right) \\
\end{array}$ \\
\hline $\begin{array}{c}\mathrm{CO}+0,5 \mathrm{O}_{2}=\mathrm{CO}_{2} \\
-\mathrm{Q}_{4}\left(-104,6 \cdot 10^{3} \mathrm{~kJ} / \mathrm{kmol}\right) \\
\end{array}$ & $\begin{array}{c}\mathrm{CO}+0,5 \mathrm{O}_{2}=\mathrm{CO}_{2} \\
-\mathrm{Q}_{18}\left(-104,6 \cdot 10^{3} \mathrm{~kJ} / \mathrm{kmol}\right)\end{array}$ \\
\hline $\begin{array}{c}\mathrm{H}_{2}+0,5 \mathrm{O}_{2}=\mathrm{H}_{2} \mathrm{O} \\
-\mathrm{Q}_{5}\left(-259 \cdot 10^{3} \mathrm{~kJ} / \mathrm{kmol}\right) \\
\end{array}$ & $\begin{array}{c}\mathrm{H}_{2}+0,5 \mathrm{O}_{2}=\mathrm{H}_{2} \mathrm{O} \\
-\mathrm{Q}_{19}\left(-259 \cdot 10^{3} \mathrm{~kJ} / \mathrm{kmol}\right) \\
\end{array}$ \\
\hline $\begin{array}{c}\mathrm{CO}_{2}=\mathrm{CO}+0,5 \mathrm{O}_{2} \\
+\mathrm{Q}_{6}\left(+330 \cdot 10^{3} \mathrm{~kJ} / \mathrm{kmol}\right)\end{array}$ & $\begin{array}{c}\mathrm{CO}_{2}=\mathrm{CO}+0,5 \mathrm{O}_{2} \\
+\mathrm{Q}_{20}\left(+330 \cdot 10^{3} \mathrm{~kJ} / \mathrm{kmol}\right) \\
\end{array}$ \\
\hline $\begin{array}{c}\mathrm{C}_{8} \mathrm{H}_{10}+10,5 \mathrm{O}_{2}=8 \mathrm{CO}_{2}+5 \mathrm{H}_{2} \mathrm{O} \\
-\mathrm{Q}_{7}\left(-1364 \cdot 10^{3} \mathrm{~kJ} / \mathrm{kmol}\right) \\
\end{array}$ & $\begin{array}{c}\mathrm{C}_{8} \mathrm{H}_{10}+10,5 \mathrm{O}_{2}=8 \mathrm{CO}_{2}+5 \mathrm{H}_{2} \mathrm{O} \\
-\mathrm{Q}_{21}\left(-1364 \cdot 10^{3} \mathrm{~kJ} / \mathrm{kmol}\right) \\
\end{array}$ \\
\hline $\begin{array}{c}\mathrm{C}_{3} \mathrm{H}_{8}+5 \mathrm{O}_{2}=3 \mathrm{CO}_{2}+4 \mathrm{H}_{2} \mathrm{O} \\
-\mathrm{Q}_{8}\left(-1575 \cdot 10^{3} \mathrm{~kJ} / \mathrm{kmol}\right) \\
\end{array}$ & $\begin{array}{c}\mathrm{C}_{3} \mathrm{H}_{8}+5 \mathrm{O}_{2}=3 \mathrm{CO}_{2}+4 \mathrm{H}_{2} \mathrm{O} \\
-\mathrm{Q}_{22}\left(-1575 \cdot 10^{3} \mathrm{~kJ} / \mathrm{kmol}\right)\end{array}$ \\
\hline $\begin{array}{c}\mathrm{C}_{4} \mathrm{H}_{10}+6,5 \mathrm{O}_{2}=4 \mathrm{CO}_{2}+5 \mathrm{H}_{2} \mathrm{O} \\
-\mathrm{Q}_{9}\left(-2150 \cdot 10^{3} \mathrm{~kJ} / \mathrm{kmol}\right)\end{array}$ & $\begin{array}{c}\mathrm{C}_{4} \mathrm{H}_{10}+6,5 \mathrm{O}_{2}=4 \mathrm{CO}_{2}+5 \mathrm{H}_{2} \mathrm{O} \\
-\mathrm{Q}_{23}\left(-2150 \cdot 10^{3} \mathrm{~kJ} / \mathrm{kmol}\right)\end{array}$ \\
\hline $\begin{array}{c}\mathrm{NO}+\mathrm{CO}=\mathrm{CO}_{2}+0,5 \mathrm{~N}_{2} \\
-\mathrm{Q}_{10}\left(-373 \cdot 10^{3} \mathrm{~kJ} / \mathrm{kmol}\right) \\
\end{array}$ & $\begin{array}{c}\mathrm{NO}+\mathrm{CO}=\mathrm{CO}_{2}+0,5 \mathrm{~N}_{2} \\
-\mathrm{Q}_{24}\left(-373 \cdot 10^{3} \mathrm{~kJ} / \mathrm{kmol}\right) \\
\end{array}$ \\
\hline $\begin{array}{l}\mathrm{NO}_{2}+2 \mathrm{CO}=2 \mathrm{CO}_{2}+0,5 \mathrm{~N}_{2} \\
-Q_{11}\left(-600 \cdot 10^{3} \mathrm{~kJ} / \mathrm{kmol}\right)\end{array}$ & $\begin{array}{l}\mathrm{NO}_{2}+2 \mathrm{CO}=2 \mathrm{CO}_{2}+0,5 \mathrm{~N}_{2} \\
-Q_{25}\left(-600 \cdot 10^{3} \mathrm{~kJ} / \mathrm{kmol}\right)\end{array}$ \\
\hline $\begin{array}{c}\mathrm{CH}_{4}=+2 \mathrm{H}_{2} \\
+\mathrm{Q}_{12}\left(+75,4 \cdot 10^{3} \mathrm{~kJ} / \mathrm{kmol}\right) \\
\end{array}$ & (2) \\
\hline $\begin{array}{c}2 \mathrm{CO}=\mathrm{C}+\mathrm{CO}_{2} \\
-\mathrm{Q}_{13}\left(-172 \cdot 10^{3} \mathrm{~kJ} / \mathrm{kmol}\right) \\
\end{array}$ & - \\
\hline $\begin{array}{c}\mathrm{CO}+\mathrm{H}_{2} \mathrm{O}=\mathrm{CO}_{2}+2 \mathrm{H}_{2} \\
-\mathrm{Q}_{14}\left(-41,2 \cdot 10^{3} \mathrm{~kJ} / \mathrm{kmol}\right)\end{array}$ & - \\
\hline
\end{tabular}

For the description of the reaction rates, the dependencies presented in Table 2 are used.
Table 2

The equations of reaction rates, depending on the components and the place of behavior

\begin{tabular}{|c|c|}
\hline On the catalyst surface & In the gas flow \\
\hline$W_{1}\left(C_{4}\right)^{\{1 C\}}=k^{\{1 C\}} \cdot C_{C H_{4}}^{\alpha\{1 C\}} \cdot C_{O_{2}}^{\beta\{1 C\}}$ & $W_{1}\left(\mathrm{CH}_{4}\right)^{\{1 V\}}=k^{\{1 V\}} \cdot C_{\mathrm{CH}_{4}}^{\alpha\{1 V\}} \cdot C_{O_{2}}^{\beta\{1 V\}}$ \\
\hline$W_{2}\left(C_{4}\right)^{\{2 C\}}=k^{\{2 C\}} \cdot C_{C H_{4}}^{\alpha\{2 C\}} \cdot C_{O_{2}}^{\beta\{2 C\}}$ & $W_{2}\left(C_{4}\right)^{\{2 V\}}=k^{\{2 V\}} \cdot C_{C_{4}}^{\alpha\{2 V\}} \cdot C_{O_{2}}^{\beta\{2 V\}}$ \\
\hline$W_{3}\left(C_{4}\right)^{\{3 C\}}=k^{\{3 C\}} \cdot C_{C H_{4}}^{\alpha\{3 C\}} \cdot C_{H_{2} O}^{\beta\{3 C\}}$ & $W_{3}\left(C_{4}\right)^{\{3 V\}}=k^{\{3 V\}} \cdot C_{C H_{4}}^{\alpha\{3 V\}} \cdot C_{H_{2} O}^{\beta\{3 V\}}$ \\
\hline$W_{4}(C O)^{\{4 C\}}=k^{\{4 C\}} \cdot C_{C O}^{\alpha\{4 C\}} \cdot C_{O_{2}}^{\beta\{4 C\}}$ & $W_{4}(C O)^{\{4 V\}}=k^{\{4 V\}} \cdot C_{C O}^{\alpha\{4 V\}} \cdot C_{O_{2}}^{\beta\{4 V\}}$ \\
\hline$W_{5}\left(H_{2}\right)^{\{5 C\}}=k^{\{5 C\}} \cdot C_{H_{2}}^{\alpha\{5 C\}} \cdot C_{O_{2}}^{\beta\{5 C\}}$ & $W_{5}\left(H_{2}\right)^{\{5 V\}}=k^{\{5 V\}} \cdot C_{H_{2}}^{\alpha\{5 V\}} \cdot C_{O_{2}}^{\beta\{5 V\}}$ \\
\hline$W_{6}\left(C_{2}\right)^{\{6 C\}}=k^{\{6 C\}} \cdot C_{C O_{2}}^{\alpha\{6 C\}}$ & $W_{6}\left(C_{2}\right)^{\{6 V\}}=k^{\{6 V\}} \cdot C_{C O_{2}}^{\alpha\{6 V\}}$ \\
\hline$W_{7}\left(C_{8} H_{10}\right)^{\{\tau C\}}=k^{\{7 C\}} \cdot C_{C_{8} H_{10}}^{\alpha\{\tau C} \cdot C_{O_{2}}^{\beta\{\tau C\}}$ & $W_{7}\left(C_{8} H_{10}\right)^{\{7 V\}}=k^{\{7 V\}} \cdot C_{C_{8} H_{10}}^{\alpha\{f V\}} \cdot C_{O_{2}}^{\beta\{T V\}}$ \\
\hline$W_{8}\left(C_{3} H_{8}\right)^{\{8 C\}}=k^{\{8 C\}} \cdot C_{C_{3} H_{8}}^{\alpha\{8 C\}} \cdot C_{O_{2}}^{\beta\{8 C\}}$ & $W_{8}\left(C_{3} H_{8}\right)^{\{8 V\}}=k^{\{8 V\}} \cdot C_{C_{3} H_{8}}^{\alpha\{8 V\}} \cdot C_{O_{2}}^{\beta\{\delta V\}}$ \\
\hline$W_{9}\left(C_{4} H_{10}\right)^{\{9 C\}}=k^{\{9 C\}} \cdot C_{C_{4} H_{10}}^{\alpha\{9 C} \cdot C_{O_{2}}^{\beta\{9 C\}}$ & $W_{9}\left(C_{4} H_{10}\right)^{\{9 V\}}=k^{\{9 V\}} \cdot C_{\left.C_{4} H_{10}\right\}}^{\alpha\{9 V} \cdot C_{O_{2}}^{\beta\{9 V\}}$ \\
\hline$W_{10}(N O)^{\{10 C\}}=k^{\{10 C\}} \cdot C_{N O}^{\alpha\{10 C\}} \cdot C_{C O}^{\beta\{10 C\}}$ & $W_{10}(N O)^{\{10 V\}}=k^{\{10 V\}} \cdot C_{N O}^{\alpha\{10 V\}} \cdot C_{C O}^{\beta\{10 V\}}$ \\
\hline$W_{11}\left(N_{2}\right)^{\{11 C\}}=k^{\{11 C\}} \cdot C_{\mathrm{NO}_{2}}^{\alpha\{11 C\}} \cdot C_{C O}^{\beta\{11 C\}}$ & $W_{11}\left(N O_{2}\right)^{\{11 V\}}=k^{\{11 V\}} \cdot C_{N_{2}}^{\alpha\{11 V\}} \cdot C_{C O}^{\beta\{11 V\}}$ \\
\hline
\end{tabular}

In Table $2 W_{(1,2, i)}^{\{i C\}}$ - reaction rate of the corresponding component on the catalyst surface, $\mathrm{kmol} /\left(\mathrm{m}^{3} \cdot \mathrm{s}\right) ; W_{(1,2 \ldots i)}^{\{i V\}}-$ reaction rate of the corresponding component in the volume of the gas flow, $\mathrm{kmol} /\left(\mathrm{m}^{3} \cdot \mathrm{s}\right) ; k^{\{i C\}}$ - the rate constant of the chemical reaction of the corresponding component on the surface of the catalyst; $k^{\{i V\}}$ - the rate constant of the chemical reaction of the corresponding component in the volume of the gas stream, $\mathrm{kmol} /\left(\mathrm{m}^{3} \cdot \mathrm{s}\right) ; C^{i\{i C\}}$ - the concentration of the corresponding component on the surface of the catalyst, $\mathrm{kmol} /\left(\mathrm{m}^{3} \cdot \mathrm{s}\right)$; $C^{\left.i i i_{V}\right\}}$ - the concentration of the corresponding component in the volume of the gas flow, $\mathrm{kmol} /\left(\mathrm{m}^{3} \cdot \mathrm{s}\right)$.

To approximate the results of studying the reaction kinetics, the structure of the Arrhenius formula is used:

$$
k^{N_{p}}=A^{N_{p}} \cdot e^{\left(\frac{-E^{N_{p}}}{R \cdot t}\right)}
$$

where $A^{N_{p}}$ - the pre-exponential factor; $E^{N_{p}}$ - activation energy of the reaction $\mathrm{N}, \mathrm{kJ} / \mathrm{mol} ; R$ - the universal gas constant, $\mathrm{kJ} /(\mathrm{kmol} \cdot \mathrm{K})$; $\mathrm{t}$ - the temperature, $\mathrm{K}$.

The material balance for the core of the flow has a mixed character and is described by (2)-(13)

$$
\begin{aligned}
& \frac{d G_{C H_{4}}}{d X}=-S_{c} \cdot W_{1 C H_{4}}^{\{1 C\}}-S_{c} \cdot W_{2 C H_{4}}^{\{2 C\}}-S_{c} \cdot W_{3 C H_{4}}^{\{3 C\}}- \\
& -S_{v} \cdot W_{1 C H_{4}}^{\{1 V\}}-S_{v} \cdot W_{2 C H_{4}}^{\{2 V\}}-S_{v} \cdot W_{3 C H_{4}}^{\{3 V\}} \cdot \\
& \frac{d G_{O}}{d X}=-S_{c} \cdot W_{4 C O}^{\{4 C\}}-S_{v} \cdot W_{4 C O}^{\{4 V\}}- \\
& -S_{c} \cdot W_{10 N O}^{\{10 C\}}-S_{v} \cdot W_{10 N O}^{\{10 V\}}-S_{c} \cdot W_{11 N O_{2}}^{\{11 C\}}- \\
& -S_{v} \cdot W_{11 N O_{2}}^{\{11 V\}}-S_{c} \cdot W_{2 C H_{4}}^{\{2 C\}}- \\
& -S_{v} \cdot W_{2 C H_{4}}^{\{2 V\}}-S_{c} \cdot W_{3 C H_{4}}^{\{3 C\}}-S_{v} \cdot W_{3 C H_{4}}^{\{3 V\}} \cdot \\
& \frac{d G_{H_{2}}}{d X}=-S_{c} \cdot W_{5 H_{2}}^{\{5 C\}}-S_{v} \cdot W_{5 H_{2}}^{\{5 V\}} \cdot \\
& \frac{d G_{C O_{2}}}{d X}=-S_{c} \cdot W_{6 C O_{2}}^{\{6 C\}}-S_{v} \cdot W_{6 C O_{2}}^{\{6 V\}} .
\end{aligned}
$$




$$
\begin{aligned}
& \frac{d G_{C_{8} H_{10}}}{d X}=-S_{c} \cdot W_{7_{C_{8} H_{10}}}^{\{7 C\}}-S_{v} \cdot W_{C_{C_{8} H_{10}}}^{\{7 \mathrm{~V}\}} \cdot \\
& \frac{d G_{C_{3} H_{8}}}{d X}=-S_{c} \cdot W_{8_{C_{3} H_{8}}}^{\{8 C\}}-S_{v} \cdot W_{8_{C_{3} H_{8}}}^{\{8 V\}} \cdot \\
& \frac{d G_{C_{4} H_{10}}}{d X}=-S_{c} \cdot W_{9_{C_{4} H_{10}}}^{\{9 C\}}-S_{v} \cdot W_{9_{C_{4} H 10}}^{\{9 V \mathrm{~V}\}} \cdot \\
& \frac{d G_{N O}}{d X}=-S_{c} \cdot W_{10 N O}^{\{10 C\}}-S_{v} \cdot W_{10 N O}^{\{10 V\}} \cdot \\
& \frac{d G_{N O_{2}}}{d X}=-S_{c} \cdot W_{11 N O_{2}}^{\{11 C\}}-S_{v} \cdot W_{11 N O_{2}}^{\{11 V\}} \cdot \\
& \frac{d G_{N_{2}}}{d X}=0,5 \cdot S_{c} \cdot W_{10 N O}^{\{10 C\}}+0,5 \cdot S_{v} \cdot W_{11 N O_{2}}^{\{11 V\}}+ \\
& +0,5 \cdot S_{v} \cdot W_{10 N O}^{\{10 V\}}+0,5 \cdot S_{c} \cdot W_{11 N O_{2}}^{\{11 C\}} \cdot \\
& S_{c}=\pi \cdot R_{C}{ }^{2} \cdot\left(1-\varepsilon_{c}\right) . \\
& S_{v}=\pi \cdot R_{C}{ }^{2} \cdot \varepsilon_{c},
\end{aligned}
$$

where $S_{c}$ - specific surface area of the solid catalyst, $\mathrm{m}^{2} ; S_{v}-$ free area of the catalyst, $\mathrm{m}^{2} ; \varepsilon_{c}-$ porosity, $\% ; R_{c}$ - the radius of the catalyst section, $m$.

The consumption of the components of the mixture at an arbitrary instant of time is determined by (14)-(17)

$$
\begin{aligned}
& G_{N_{i}}=G_{N O_{0}}-2 \cdot\left(G_{N_{2 i}}-G_{N_{20}}\right)-\left(G_{N O_{2 i}}-G_{N O_{20}}\right) \text {. } \\
& G_{C O_{2 i}}=G_{C_{20}}-\left(\frac{W_{1 C H_{4}}^{\{1 C\}}}{W_{1 H_{4}}^{\{1 C\}}+W_{2 \mathrm{CH}_{4}}^{\{2 C\}}+W_{3 C H_{4}}^{\{3 C\}}}\right) \times \\
& \times\left(G_{C_{4 i}}-G_{C H_{40}}\right)-2 \cdot\left(G_{C O_{i}}-G_{C O_{0}}\right)- \\
& -8 \cdot\left(G_{C_{8} H_{10 i}}-G_{C_{8} H_{100}}\right)-3 \cdot\left(G_{C_{3} H_{8 i}}-G_{C_{3} H_{80}}\right)- \\
& -4 \cdot\left(G_{C_{4} H_{10 i}}-G_{C_{4} H_{100}}\right) \text {. } \\
& G_{O_{2 i}}=G_{O_{20}}-2 \cdot\left(\frac{W_{1 C H_{4}}^{\{1 C\}}}{W_{1 C H_{4}}^{\{1 C\}}+W_{2 C H_{4}}^{\{2 C\}}+W_{3 C H_{4}}^{\{3 C\}}}\right) \cdot\left(G_{C_{4 i}}-G_{C H_{40}}\right)- \\
& \left(\frac{W_{2 C H_{4}}^{\{2 C\}}}{W_{1 C H_{4}}^{\{1 C\}}+W_{2 C H_{4}}^{\{2 C\}}+W_{3 C H_{4}}^{\{3 C\}}}\right) \cdot\left(G_{C_{4 i}}-G_{C_{40}}\right)- \\
& -0,5 \cdot\left(G_{C O_{i}}-G_{C O_{0}}\right)-0,5 \cdot\left(G_{H_{2 i}}-G_{H_{20}}\right) \\
& -10,5 \cdot\left(G_{C_{8} H_{10 i}}-G_{C_{8} H_{100}}\right)-3 \cdot\left(G_{C_{3} H_{8 i}}-G_{C_{3} H_{80}}\right)- \\
& -4 \cdot\left(G_{C_{4} H_{10 i}}-G_{C_{4} H_{100}}\right)+\left(G_{N_{2 i}}-G_{N_{20}}\right)-0,5 \cdot\left(G_{N_{2 i}}-G_{N_{20}}\right) \text {. } \\
& G_{\mathrm{H}_{2} \mathrm{O}_{i}}=G_{\mathrm{H}_{2} \mathrm{O}_{0}}-3 \cdot\left(G_{C_{3} H_{8 i}}-G_{C_{3} H_{80}}\right)- \\
& -4 \cdot\left(G_{C_{4} H_{10 i}}-G_{C_{4} H_{100}}\right)- \\
& -5 \cdot\left(G_{C_{8} H_{10 i}}-G_{C_{8} H_{100}}\right)- \\
& -4 \cdot\left(\frac{W_{3 \mathrm{CH}_{4}}^{\{3 \mathrm{C}\}}}{W_{1 \mathrm{CH}_{4}}^{\{1 \mathrm{C}\}}+W_{2 \mathrm{CH}_{4}}^{\{2 C\}}+W_{3 C H_{4}}^{\{3 C\}}}\right) \cdot\left(G_{\mathrm{CH}_{4 i}}-G_{\mathrm{CH}_{40}}\right) \text {. }
\end{aligned}
$$

The balance of mass transfer between the core of the flow and the surface of the catalyst, taking into account the stoichiometry (18)-(28)

$$
\begin{aligned}
& g_{\mathrm{CH}_{4}} \cdot S_{s}=S_{s} \cdot \beta_{\mathrm{CH}_{4}} \cdot\left(C_{\mathrm{CH}_{4}}-C_{\mathrm{CH}_{4}}^{*}\right)= \\
& =W_{1 \mathrm{CH}_{4}}^{\{1 \mathrm{C}\}}+W_{2 \mathrm{CH}_{4}}^{\{2 \mathrm{C}\}}+W_{3 \mathrm{CH}_{4}}^{\{3 \mathrm{C}\}} \text {. } \\
& g_{C O} \cdot S_{s}=S_{s} \cdot \beta_{C O} \cdot\left(C_{C O}-C_{C O}^{*}\right)= \\
& =W_{10 \mathrm{NO}}^{\{10 C\}}+W_{4 C O}^{\{4 C\}}-W_{6 C O_{2}}^{\{6 C\}}-W_{2 \mathrm{CH}_{4}}^{\{2 C\}}-W_{3 \mathrm{CH}_{4}}^{\{3 C\}} . \\
& g_{O_{2}} \cdot S_{s}=S_{s} \cdot \beta_{O_{2}} \cdot\left(C_{O_{2}}-C_{O_{2}}^{*}\right)= \\
& =0,5 \cdot W_{4 C O}^{\{4 C\}}+2 \cdot W_{1 C H_{4}}^{\{1 C\}}+W_{2 C H_{4}}^{\{2 C\}}+ \\
& +0,5 W_{5 H_{2}}^{\{5 C\}}+10,5 \cdot W_{7 C_{8} H_{10}}^{\{7 C\}}+ \\
& +5 \cdot W_{8 C_{3} H_{8}}^{\{8 C\}}+6,5 \cdot W_{9 C_{4} H_{10}}^{\{9 C\}} \text {. } \\
& g_{H_{2}} \cdot S_{s}=S_{s} \cdot \beta_{H_{2}} \cdot\left(C_{H_{2}}-C_{H_{2}}^{*}\right)= \\
& =W_{5 \mathrm{H}_{2}}^{\{5 \mathrm{C}\}}-W_{2 \mathrm{CH}_{4}}^{\{2 \mathrm{C}\}}-3 \cdot W_{3 \mathrm{CH}_{4}}^{\{3 \mathrm{C}\}} \text {. } \\
& g_{C_{8} H_{10}} \cdot S_{s}=S_{s} \cdot \beta_{C_{8} H_{10}} \cdot\left(C_{C_{8} H_{10}}-C_{C_{8} H_{10}}^{*}\right)=W_{7 C_{8} H_{10}}^{\{7 C\}} . \\
& g_{C_{3} H_{8}} \cdot S_{s}=S_{s} \cdot \beta_{C_{3} H_{8}} \cdot\left(C_{C_{3} H_{8}}-C_{C_{3} H_{8}}^{*}\right)=W_{8 C_{3} H_{8}}^{\left\{8 C_{8}\right.} \text {. } \\
& g_{C_{4} H_{10}} \cdot S_{s}=S_{s} \cdot \beta_{C_{4} H_{10}} \cdot\left(C_{C_{4} H_{10}}-C_{C_{4} H_{10}}^{*}\right)=W_{9 C_{4} H_{10}}^{\left\{9 C_{10}\right.} . \\
& g_{N O} \cdot S_{s}=S_{s} \cdot \beta_{N O} \cdot\left(C_{N O}-C_{N O}^{*}\right)=W_{10 N O}^{\{10 C\}} \text {. } \\
& g_{N_{2}}=-0,5 \cdot g_{N O} \text {. } \\
& g_{\mathrm{CO}_{2}}=-g_{\mathrm{CO}}-8 \cdot g_{\mathrm{C}_{8} \mathrm{H}_{10}}- \\
& -3 \cdot g_{C_{3} H_{8}}-4 \cdot g_{C_{4} H 10}-g_{H_{2}} \text {. } \\
& g_{H_{2} \mathrm{O}}=-5 \cdot g_{C_{8} H_{10}}-4 \cdot g_{C_{3} H_{8}}-5 \cdot g_{C_{4} H 10}- \\
& -2 \cdot g_{\mathrm{CH} 4} \cdot\left(\frac{W_{1 C H_{4}}^{\{1 C\}}}{W_{1 H_{4}}^{\{1 C\}}+W_{2 \mathrm{CH}_{4}}^{\left\{2 C_{4}\right.}+W_{3 \mathrm{CH}_{4}}^{\{3 C\}}}\right)- \\
& -g_{C H 4} \cdot\left(\frac{W_{2 C H_{4}}^{\{2 C\}}}{W_{1 C H_{4}}^{\{1 C\}}+W_{2 C H_{4}}^{\{2 C\}}+W_{3 C H_{4}}^{\{3 C\}}}\right)= \\
& =-5 \cdot g_{C_{8} H_{10}}-4 \cdot g_{C_{3} H_{8}}-5 \cdot g_{C_{4} H 10}- \\
& -2 \cdot S_{1} \cdot g_{\mathrm{CH}_{4}}-S_{2} \cdot g_{\mathrm{CH}_{4}},
\end{aligned}
$$

where $g_{i}$ - the diffusion flux density, $\mathrm{kmol} /\left(\mathrm{m}^{2} \cdot \mathrm{s}\right) ; \beta_{i}-$ the mass transfer coefficient, $\mathrm{m} / \mathrm{s} ; S_{i}-$ the selectivity of the methane oxidation reaction along one of the directions.

The energy balance in the gas phase has the form (29)

$$
\begin{gathered}
G_{i} \cdot c_{i}^{p} \cdot \frac{d T}{d x}=F \cdot\left(1-\varepsilon_{c}\right) \cdot S_{s} \times \\
\sum_{i} \times\left[\begin{array}{l}
\left.\alpha_{T} \cdot(\Theta-T)+\sum_{i} g_{i} \cdot\left(I_{i}^{\prime}-I_{i}(T)\right)-\right]+ \\
-2 \cdot k_{p} \cdot \sigma \cdot\left(2 \cdot T^{4}-T_{e}^{4}\right)
\end{array}\right] \\
+F \cdot \varepsilon_{c} \cdot\left(\begin{array}{l}
W_{1 C H_{4}}^{\{1 \mathrm{~V}\}} \cdot Q_{1}+W_{2 C H_{4}}^{\{2 \mathrm{~V}\}} \cdot Q_{2}+W_{3 C H_{4}}^{\{3 \mathrm{~V}\}} \cdot\left(-Q_{3}\right)+ \\
+W_{4 C O}^{\{4 \mathrm{~V}\}} \cdot Q_{4}+W_{5 H_{2}}^{\{5 \mathrm{~V}\}} \cdot Q_{5}+W_{6 C O_{2}}^{\{6 \mathrm{~V}\}} \cdot\left(-Q_{6}\right)+ \\
+W_{7 C_{8} H_{10}}^{\{\mathrm{V}\}} \cdot Q_{7}+W_{8 C_{3} \mathrm{H}_{8}}^{\{8 \mathrm{~V}\}} \cdot Q_{8}+ \\
+W_{9 C_{4} \mathrm{H}_{10}}^{\{9 \mathrm{~V}} \cdot Q_{9}+W_{10 \mathrm{NO}}^{\{10 \mathrm{~V}\}} \cdot Q_{10}
\end{array}\right),
\end{gathered}
$$

where $\alpha_{T}-$ the heat transfer coefficient for the interfacial surface, $\mathrm{W} /\left(\mathrm{m}^{2} \cdot \mathrm{K}\right) ; I_{i}$ - the enthalpy of the $i$-th component of the gas flow, $\mathrm{kJ} / \mathrm{kmol}$. 
The energy balance in the solid phase under the condition of continuity of the heat flux through the phase interface and taking into account the thermal losses (30)

$\rho_{c} \cdot C_{c} \cdot \frac{\partial \Theta}{\partial \tau}=$

$=S_{s} \cdot\left[\alpha_{T} \cdot(T-\Theta)+\lambda_{c} \cdot \frac{\partial^{2} \Theta}{\partial x^{2}}-\sum_{i} g_{i} \cdot I_{i}-\sigma \cdot \xi \cdot\left(\Theta^{4}-T_{e}^{4}\right)\right]+$

$+W_{1 C H_{4}}^{\{1 C\}} \cdot Q_{1}+W_{2 C H_{4}}^{\{2 C\}} \cdot Q_{2}+W_{3 C H_{4}}^{\{3 C\}} \cdot\left(-Q_{3}\right)+$

$+W_{4 C O}^{\{4 C\}} \cdot Q_{4}+W_{5 H_{2}}^{\{5 C\}} \cdot Q_{5}+W_{6 C O_{2}}^{\{5 C\}} \cdot\left(-Q_{6}\right)+$

$+W_{7 C_{8} H_{10}}^{\{7 C\}} \cdot Q_{7}+W_{8 C_{3} H_{8}}^{\{8 C\}} \cdot Q_{8}+W_{9 C_{4} H_{10}}^{\{9 C\}} \cdot Q_{9}+$

$+W_{10 N O}^{\{10 C\}} \cdot Q_{10}-\frac{2}{R_{c} \cdot\left(1-\varepsilon_{c}\right) \cdot k_{e} \cdot\left(\Theta-T_{e}\right)}$,

where $k_{e}$ - the of heat transfer coefficient from the catalyst to the environment; $T_{e}$ - the ambient temperature, $\mathrm{K} ; T$ - the temperature of the gas flow, $\mathrm{K} ; \Theta$-the surface temperature of the catalyst, $\mathrm{K}$.

Critical equations are used to determine the values of mass transfer coefficients and heat transfer:

$$
\begin{aligned}
& \mathrm{Nu}=0,395 \cdot \operatorname{Re}^{0,64} \cdot \operatorname{Pr}^{0,33} ; \\
& \mathrm{Re}=30 \div 8 \cdot 10^{4} ; \operatorname{Pr}=0,6 \div 3600 \\
& \mathrm{Nu}=0,725 \cdot \mathrm{Re}^{0,47} \cdot \operatorname{Pr}^{0,43} ; \\
& \mathrm{Re}=2 \div 30 ; \mathrm{Pr}=0,6 \div 3600, \\
& \mathrm{Sh}=0,395 \cdot \operatorname{Re}^{0,64} \cdot \mathrm{Sc}^{0,33} ; \\
& \mathrm{Re}=30 \div 8 \cdot 10^{4} ; \mathrm{Sc}=0,6 \div 3600 \\
& \mathrm{Sh}=0,725 \cdot \mathrm{Re}^{0,47} \cdot \mathrm{Sc}^{0,43} ; \\
& \operatorname{Re}=2 \div 30 ; \quad \mathrm{Sc}=0,6 \div 3600, \\
& \beta_{i}=\frac{\mathrm{Sh} \cdot D_{i}}{d_{e q}} \\
& \alpha_{T}=\frac{\mathrm{Nu} \cdot \lambda_{g}}{d_{e q}}
\end{aligned}
$$

where $d_{e q}$ - the equivalent grain diameter of the catalyst, $\mathrm{m}$.

The solution of coupled differential equations is performed using a computer account on the basis of the fourth-order Runge-Kutta numerical method. Calculations are carried out in a specially developed program "Complete two-phase mathematical model of methane oxidation”.

\section{Results of mathematical modeling of the methane oxidation process on a fibrous catalyst}

As a prototype for modeling the methane oxidation process on a heterogeneous catalyst, experimental data and the actual kinetics of methane oxidation on a fiber catalyst are taken.

\begin{tabular}{|c|c|c|c|c|c|c|}
\hline \multicolumn{2}{|c|}{$\begin{array}{c}\text { Gas flow } \\
\text { temperature, } \\
T, \mathrm{~K}\end{array}$} & \multirow[t]{2}{*}{$\begin{array}{l}C_{C H 4}^{\{V\}} \cdot 10^{4}, \\
\mathrm{kmol} / \mathrm{nm}^{3}\end{array}$} & \multirow[t]{2}{*}{$\begin{array}{l}C_{\mathrm{CO} 2}^{\{V\rangle} \cdot 10^{4}, \\
\mathrm{kmol} / \mathrm{nm}^{3}\end{array}$} & \multirow[t]{2}{*}{$\begin{array}{r}r\left(\mathrm{CO}_{2}\right) \cdot 10^{3}, \\
\mathrm{kmol} / \mathrm{m}^{3} \cdot \mathrm{s}\end{array}$} & \multirow[t]{2}{*}{$\begin{array}{c}\xi\left(\mathrm{CH}_{4}\right), \\
\%\end{array}$} & \multirow[t]{2}{*}{$\begin{array}{c}w \\
\mathrm{~nm} / \mathrm{s}\end{array}$} \\
\hline$T_{\text {in }}$ & $T_{\text {out }}$ & & & & & \\
\hline 573,15 & 581,65 & 7,71 & 0,10 & 0,31 & 1,34 & 1,92 \\
\hline 583,15 & 593,05 & 7,67 & 0,15 & 0,43 & 1,857 & 1,95 \\
\hline 613,15 & 630,75 & 7,43 & 0,38 & 1,14 & 4,89 & 2,05 \\
\hline 623,15 & 645,35 & 7,28 & 0,53 & 1,58 & 5,84 & 2,09 \\
\hline 633,15 & 661,85 & 7,07 & 0,74 & 2,21 & 9,531 & 2,12 \\
\hline 663,15 & 744,25 & 5,32 & 2,50 & 7,41 & 31,96 & 2,22 \\
\hline 673,15 & 820,25 & 3,05 & 4,77 & 14,15 & 61,01 & 2,26 \\
\hline 703,15 & 927,65 & 0,23 & 7,58 & 22,51 & 97,02 & 2,36 \\
\hline 723,15 & 948,25 & 0,19 & 7,62 & 22,64 & 97,6 & 2,42 \\
\hline 773,15 & 996,85 & 0,11 & 7,70 & 22,86 & 98,56 & 2,59 \\
\hline 823,15 & 1048,4 & 0,07 & 7,74 & 22,99 & 99,12 & 2,76 \\
\hline
\end{tabular}

Catalyst characteristics: density $-\rho=0.453 \mathrm{~g} / \mathrm{cm}^{3}$; porosity $-\varepsilon=87.02 \%$; volume of the catalyst $-V=4.6 \cdot 10^{-6} \mathrm{~m}^{3}$; height of the layer $-l=30 \mathrm{~mm}$; mass $-m=1.54 \mathrm{~g}$.
The data of the experimental study of the catalyst are presented in Table 3.

Table 3

Data of an experimental study of the kinetics of methane complete oxidation on a catalyst. The methane concentration at the reactor inlet $C_{\mathrm{CH}_{4} \text { in }}^{\{V\}}=0,00078125 \mathrm{kmol} / \mathrm{nm}^{3}$

Based on the experimental data, a macrokinetic equation of the rate of complete oxidation of methane is derived, the similarity criteria, mass transfer coefficients, and heat transfer are calculated.

For the conditions of the experiment, the macrokinetic equation has the form:

$$
r=A \cdot e^{\frac{-E a}{R \cdot T}} \cdot C_{\mathrm{CH}_{4}}=8,39 \cdot 10^{9} \cdot e^{\frac{-112300}{R \cdot T}} \cdot C_{\mathrm{CH}_{4} \text { gas }} .
$$

The value of the activation energy and the pre-exponential factor is determined by integral methods. These values are typical for the methane oxidation reaction on a fibrous $\mathrm{MgAl}_{2} \mathrm{O}_{4} / \mathrm{NiO} / \mathrm{Cr}_{2} \mathrm{O}_{3}$ catalyst.

To determine the areas of the oxidation process on the fiber catalyst, the rate of methane oxidation is calculated (37). The calculation data are shown in Fig. 1.

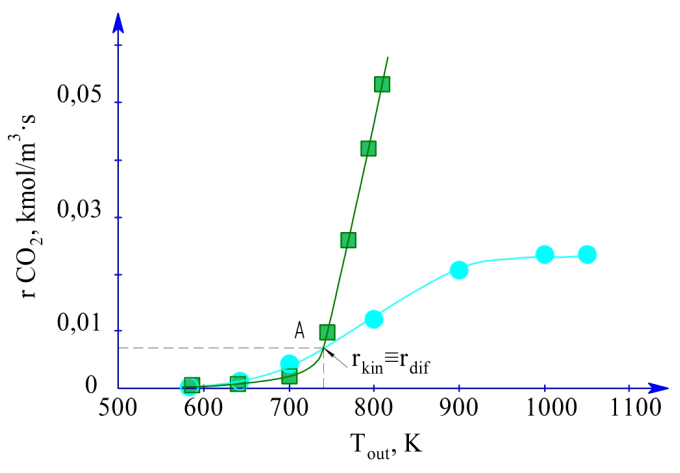

$$
\begin{gathered}
\mathrm{CO}_{2} \text { formation rate } \square \begin{array}{c}
\mathrm{CO}_{2} \text { formation rate according } \\
\text { to the macrokinetic equation }
\end{array}
\end{gathered}
$$

Fig. 1. Experimental and macrokinetic rate of methane oxidation on the catalyst

As can be seen from the graphs, the point $\mathrm{A}$ is the point of separation of the methane oxidation rate, calculated from 
the macrokinetic equation, and the experimental kinetic velocity. At a flue gas temperature of $720-750 \mathrm{~K}$ and a degree of methane conversion of $30-35 \%$, a sharp increase in the chemical oxidation rate is observed, while the observed rate decreases due to the influence of external diffusion factors. Therefore, it is possible to conclude that the further conversion of methane does not depend on chemical kinetics and point $\mathrm{A}$ is the boundary of the kinetic region. Also, point $\mathrm{A}$ is a point of the identity equality $r_{\text {kin }}-r_{\text {dif }}$.

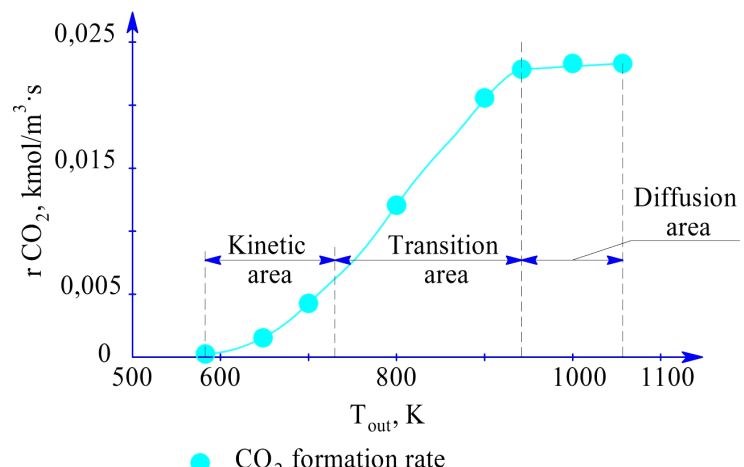

Fig. 2. Areas of heterogeneous catalytic methane oxidation process on the experimental fibrous catalyst

For the numerical analysis of the oxidation process, the values of similarity criteria, mass coefficients and heat transfer are calculated, which are within the following limits: Nusselt number $-\mathrm{Nu}=2.01-2.06$; Prandtl number is $\operatorname{Pr}=0.67-0.70$; Reynolds criterion is $\mathrm{Re}=10.3$; Sherwood's criterion is $\mathrm{Sh}=0.72-0.57$; $\mathrm{Schmidt}$ number is $\mathrm{Sc}=0.06-0.036$. The mass-transfer coefficient averaged $-\beta=0,4-0,95 \mathrm{~m} / \mathrm{s}$, the heat transfer coefficient is $\alpha=280-650 \mathrm{~W} / \mathrm{m}^{2} \cdot \mathrm{K}$.

The main aim of the modeling is determination of the methane concentration on the catalyst surface, the surface temperature of the catalyst surface, and the degree of conversion across the catalyst bed. The numerical modeling data are presented in Table 4.

Table 4

Results of mathematical modeling of methane oxidation process on a fibrous catalyst

\begin{tabular}{|c|c|c|c|c|c|c|c|c|}
\hline \multicolumn{4}{|c|}{ Specified temperature, $\mathrm{K}$} & \multicolumn{4}{|c|}{$\begin{array}{c}\text { Specified concentrations } \\
\mathrm{C}_{\mathrm{CH} 4} \cdot 10^{4} \mathrm{kmol} / \mathrm{nm}^{3}\end{array}$} & \multirow{2}{*}{$\xi, \%$} \\
\hline$T_{\text {in }}$ & $T_{\text {out }}$ & $\Theta_{\text {in }}$ & $\Theta_{\text {out }}$ & $C_{\left(C H_{4}\right)}^{\{V\}\}_{i n}}$ & $C_{\left(\mathrm{CH}_{4}\right)}^{\{\mathrm{V}\} \mathrm{H}_{\mathrm{i}}}$ & $C_{\left(C H_{4}\right)}^{\{c\} i n}$ & $C_{\left(\mathrm{CH}_{4}\right)}^{\{\mathrm{C}\}_{\text {in }}}$ & \\
\hline \multicolumn{9}{|c|}{ Kinetic area } \\
\hline 603 & 605 & 608 & 615 & 4,09 & 4,04 & 3,72 & 3,66 & 1,4 \\
\hline 613 & 620 & 618 & 629 & 4,31 & 4,24 & 3,51 & 3,43 & 1,9 \\
\hline 643 & 655 & 653 & 672 & 4,34 & 4,14 & 3,48 & 3,30 & 5,1 \\
\hline 653 & 662 & 663 & 691 & 4,39 & 4,15 & 3,42 & 3,21 & 6,1 \\
\hline 663 & 678 & 678 & 712 & 4,45 & 4,05 & 3,37 & 3,03 & 10 \\
\hline 693 & 729 & 708 & 780 & 4,60 & 3,23 & 3,22 & 2,13 & 33,6 \\
\hline \multicolumn{9}{|c|}{ Transition and diffusion areas } \\
\hline 703 & 775 & 718 & 841 & 4,65 & 2,03 & 3,16 & 1,13 & 64,1 \\
\hline 743 & 840 & 763 & 875 & 4,78 & 0,67 & 3,03 & 0,065 & 97,8 \\
\hline 763 & 878 & 783 & 922 & 4,87 & 0,63 & 2,95 & 0,032 & 98,9 \\
\hline 813 & 925 & 833 & 994 & 5,06 & 0,61 & 2,76 & 0,0027 & 99,9 \\
\hline 873 & 998 & 893 & 1085 & 5,22 & 0,63 & 2,59 & 0,00259 & 99,9 \\
\hline
\end{tabular}

Fig. 3 shows a comparison of the values of methane concentrations in the gas flow and the temperature of the off-gases after passing the catalyst, obtained experimentally and numerically.
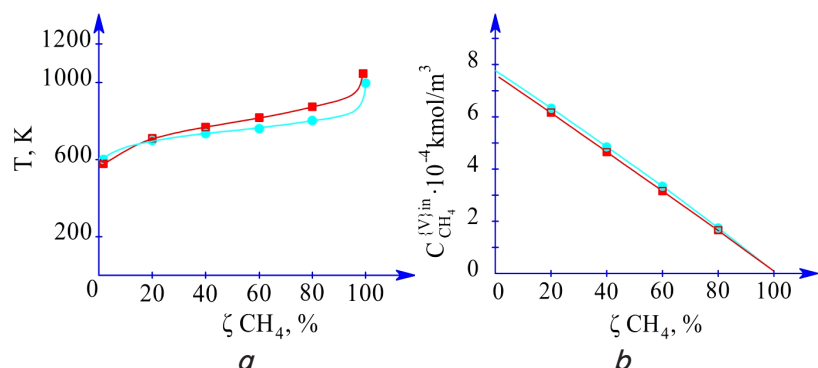

Estimated methane concentration in the gas flow and temperature of the exhaust gases after passage of the catalyst, $\mathrm{kmol} / \mathrm{m}^{3}, \mathrm{~K}$

- Experimental concentration of methane in the gas flow of exhaust gases after passing the catalyst, $\mathrm{kmol} / \mathrm{m}^{3}, \mathrm{~K}$

Fig. 3. Comparison of the values of: $a-$ the experimental and calculated temperature of the gas flow heating, $b-$ the experimental and calculated methane concentration in the gas flow

Analysis of modeling results and their comparison with experimental data confirm the correctness of the calculation algorithm.

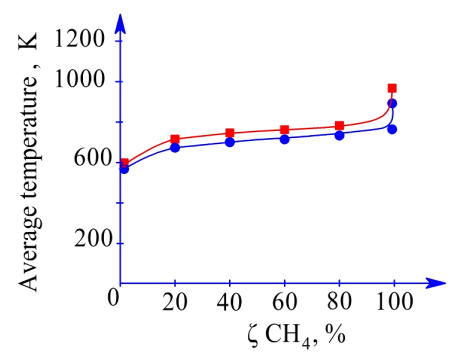

Average calculated value of the temperature

- of the heated gas flow, K

- Average calculated value of the surface temperature of the catalyst surface, $\mathrm{K}$

Fig. 4. Comparison of the average over the catalyst bed temperature of the surface heating of the catalyst and the gas flow from the methane conversion degree

As a result of calculations, it is found that the catalyst surface is superheated with respect to the gas flow, but the maximum temperature to which the catalyst warms up, namely, $1085 \mathrm{~K}$, does not exceed the allowable value of $1870 \mathrm{~K}$.

Fig. 5 shows the temperature dependence of the surface temperature of the catalyst, the temperature of the gas flow heating, the surface and volume concentrations of methane over the catalyst bed. The data are obtained by means of mathematical modeling.

Using these changes in methane concentration on the surface of the catalyst, an improved microkinetic equation of the oxidation rate is calculated with allowance for the catalyst heating temperature:

$$
r=A \cdot e^{\frac{-E a}{R \cdot T}} \cdot C_{\mathrm{CH}_{4}}=1,75 \cdot 10^{9} \cdot e^{\frac{-85413}{R \cdot \Theta_{c a t}}} \cdot C_{\mathrm{CH}_{4} \text { cat }} .
$$

As a result of refined calculations, the activation energy for the methane oxidation reaction on the catalyst is $85.4 \mathrm{~kJ} / \mathrm{kmol}$. 

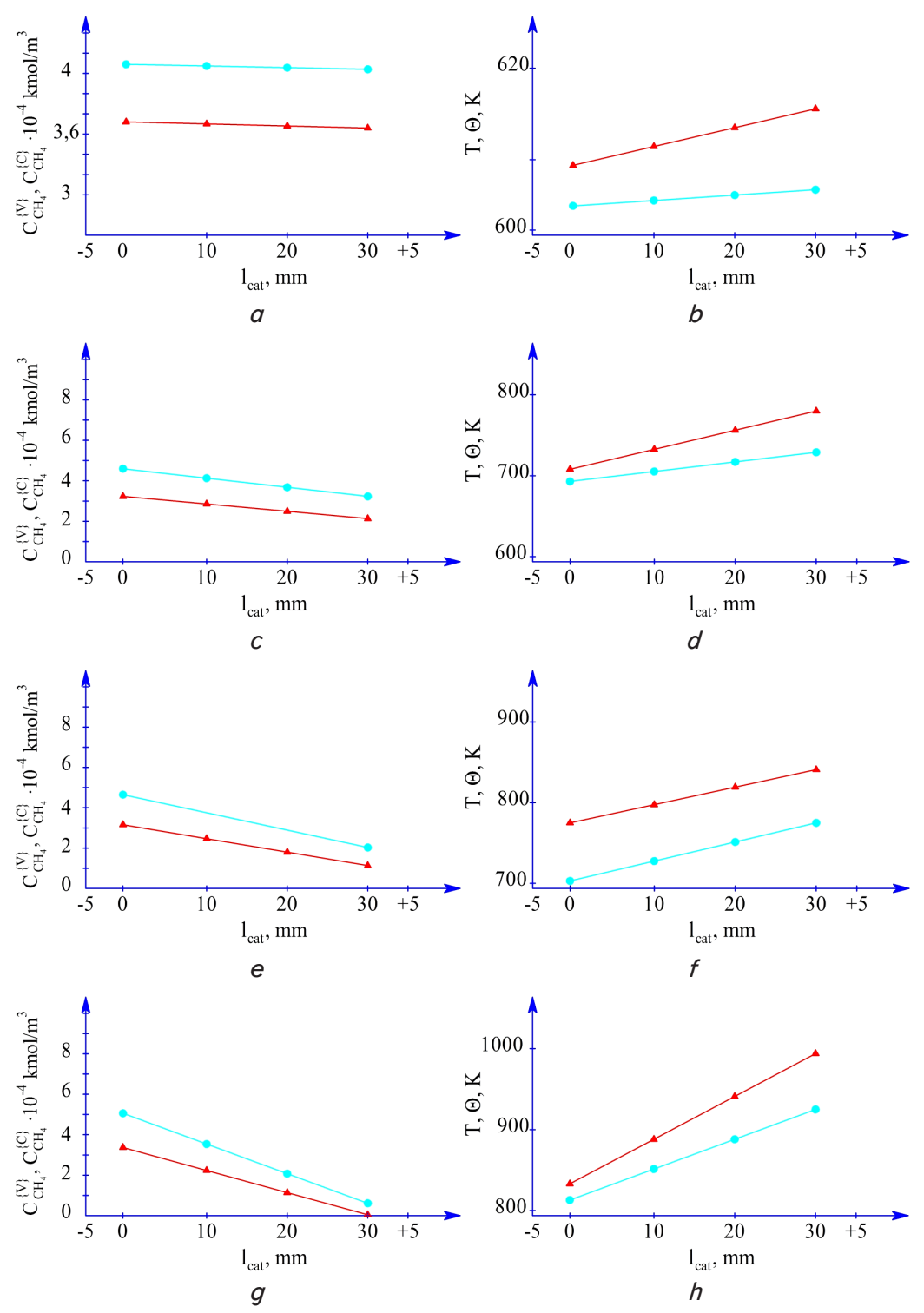

The change in methane concentration in the gas flow and the temperature

- of the gas flow, $\mathrm{kmol} / \mathrm{m}^{3}, \mathrm{~K}$

ه The change in methane concentration on the catalyst surface and the surface temperature of the catalyst surface, $\mathrm{kmol} / \mathrm{m}^{3}, \mathrm{~K}$

Fig. 5. Results of modeling the methane oxidation process on a fibrous catalyst: $a, b-$ initial temperature of the gas flow $603 \mathrm{~K}$, methane conversion $\xi-1.4 \% ; c, d-$ initial temperature of gas flow $693 \mathrm{~K}$, methane conversion $\xi-33.6 \% ; e, f-$ initial temperature of the gas flow $703 \mathrm{~K}$, methane conversion $\xi-64.1 \% ; g, h-$ initial temperature of the gas flow $813 \mathrm{~K}$, methane conversion $\xi-99.9 \%$

For the temperature of the mixture of exhaust gases, at which the identical equality of the chemical and mass exchange rates of the reaction is observed, the thermophysical properties of the gas mixture and the hydrodynamic parameters of the course of the methane oxidation reaction are determined. Density of the mixture $-\rho=0.43 \mathrm{~kg} / \mathrm{m}^{3}$; kinematic viscosity $-v=8.47 \cdot 10^{-5} \mathrm{~m}^{2} / \mathrm{s}$; methane diffusion coefficient in a mixture of gases $-D=1.9 \cdot 10^{-4} \mathrm{~m}^{2} / \mathrm{s}$; coefficient of mass transfer $-\beta=0,88 \mathrm{~m} / \mathrm{s}$; Prandtl number $-\operatorname{Pr}=0.731$; Reynolds criterion $-\mathrm{Re}=10.3$; Sherwood's criterion $-\mathrm{Sh}=1.54$; Schmidt's criterion $-\mathrm{Sc}=0.45$.

Using the identical equation $r_{k i n} \equiv r_{d i}$, the actually working (effective) surface of the catalyst is calculated.

$$
\begin{aligned}
& S_{w}=\frac{r_{k i n}}{\beta_{\mathrm{CH}_{4}} \cdot\left(C_{\mathrm{CH}_{4}}^{\text {gas }}-C_{\mathrm{CH}_{4}}^{\text {gas }}\right)}= \\
& =\frac{1,75 \cdot 10^{9} \cdot e^{\frac{-85413}{R \cdot \Theta c a t}} \cdot C_{\mathrm{CH}_{4} c a t}}{\beta_{\mathrm{CH}_{4}} \cdot\left(C_{\mathrm{CH}_{4}}^{\text {gas }}-C_{\mathrm{CH}_{4}}^{c a t}\right)} .
\end{aligned}
$$

As a result of the calculation, the effective working surface of the catalyst is calculated, which amounted to $S_{w}=7640 \mathrm{~m}^{2} / \mathrm{m}^{3}$. At the same time, the calculated specific surface calculated and presented in [11], with an average fiber diameter of $0.003 \mathrm{~mm}$, is about $20,000 \mathrm{~m}^{2} / \mathrm{m}^{3}$.

\section{Discussion of the results of the methane oxidation modeling}

A positive result of this research is the confirmation of data on the kinetics of catalytic oxidation of methane, obtained as a result of modeling by experimental data. This result not only confirms the operability of the algorithm of the mathematical model, but also makes it possible to obtain data that is problematic to obtain experimentally. For example: the dynamics of the change in the concentration of methane, both on the surface of the catalyst and in the gas stream, the temperature of the surface heating of the catalyst along the catalyst bed, determine the maximum temperature of catalyst heating. For example, at the maximum degree of methane conversion, the value of the maximum catalyst warming temperature (Fig. 4) does not exceed the value of the temperature that both the catalytic contact and the catalyst carrier withstand.

Also, the data obtained by mathematical calculation confirms the high activity of the catalyst.

In Fig. 2, the areas of methane oxidation are described. Applying the mathematical model, it is established that in the temperature range of $600-700 \mathrm{~K}$ the process proceeds in the kinetic region, in which only the chemical activity affects the rate of the process, initial concentrations and temperature. The transition region is characterized by a gradual increase in the reaction rate, to a lesser extent, depending on the concentration and temperature. In this region, the reaction rate is affected by the specific contact area, concentration, mass and heat removal, and the activation energy decreases and is about 0.5 from the activation energy in the kinetic regime. In the diffusion region, the main contribution to the rate of the reaction is exerted by mass-exchange and heat-exchange processes, and the rate of the process depends little on temperature.

Fig. 1 shows the dependence of $\mathrm{CO}_{2}$ formation rate. So, if the macrokinetic equation is used to determine the reaction rate, then the velocity values coincide with the actual experimental data in the kinetic region. For the transition region and diffusion, this equation can't be applied. Thanks 
to this study, a microkinetic equation is derived that takes into account the effect on the reaction rate of the surface temperature of the catalyst and the methane concentration on the surface.

The practical value of this research is a rapid analysis of the mechanism of methane oxidation on various catalysts without carrying out a large number of physical experiments. Modeling results can be used in the development of various catalytic burners and light heaters.

In the future, it is planned to improve the model, with the aim of using methane vapor conversion catalysts for analysis.

\section{Conclusions}

1. Hydrodynamic indices of the process in a laboratory flow reactor are determined. Nusselt number is $\mathrm{Nu}=2.01-2.06$; Prandtl number is $\mathrm{Pr}=0.67-0.70$; Reynolds criterion is
$\mathrm{Re}=10.3-13.01$; Sherwood's criterion is $\mathrm{Sh}=0.72-0.57$; Schmidt number is $\mathrm{Sc}=0.06-0.036$.

2 . With the help of calculations, the ranges of the change in the heat transfer coefficient $\left(\alpha=280-650 \mathrm{~W} / \mathrm{m}^{2} \cdot \mathrm{K}\right)$ and mass transfer $\beta=0.4-0.95 \mathrm{~m} / \mathrm{s}$.

3. Using mathematical modeling, the temperature of the catalyst surface is determined, the maximum value is $\Theta_{\max }=1085 \mathrm{~K}$, the minimum concentration of methane on the surface of $\mathrm{C}_{\mathrm{CH} 4}=1.2 \cdot 10^{-6} \mathrm{kmol} / \mathrm{m}^{3}$.

4. Due to the identical equality of the kinetic and diffusion rate of $\mathrm{CO}_{2}$ formation, an effective working surface is calculated that participates in the heterogeneously catalytic reaction of complete oxidation of methane $S_{w}=7640 \pm 150 \mathrm{~m}^{2} / \mathrm{m}^{3}$.

The experimental kinetic data of methane oxidation on the catalyst have been confirmed by mathematical calculations, a refined microkinetic equation has been derived, the flow areas of the process have been established, and the performance of the synthesized catalyst has been established.

\section{References}

1. Vernikovskaya, N. Modelirovanie protsessa okisleniya v trubchatyh elementah kataliticheskih nagrevateley [Text]/N. Vernikovskaya, A. Chasovnikova, V. Chumachenko // Himiya v interesah ustoychivogo razvitiya. - 2017. - Issue 1. - P. 5-10. doi: 10.15372/ khur20170101

2. Lashina, E. Matematicheskoe modelirovanie avtokolebaniy v reaktsii okisleniya metana na nikele: Izotermicheskaya model' [Text] / E. Lashina, V. Kaichev, N. Chumakova, V. Ustyugov, G. Chumakov, V. Buhtiyarov // Kinetika i kataliz. - 2012. - Issue 3. - P. $389-399$.

3. Paharukov, I. Issledovanie kinetiki glubokogo okisleniya metana s ispol'zovaniem usovershenstvovannogo protochno tsirkulyatsionnogo metoda [Text] / I. Paharukov, N. Bobrov, V. Parmon // Kataliz v promyshlennosti. - 2008. - Issue 6. - P. 11-16.

4. Boukhalfa, N. Chemical Kinetic Modeling of Methane Combustion [Text] / N. Boukhalfa // Procedia Engineering. - 2016. Vol. 148. - P. 1130-1136. doi: 10.1016/j.proeng.2016.06.561

5. Kazakov, D. Opisanie biokataliticheskogo okisleniya metana s ispol'zovaniem modeley mnogosubstratnoy kinetiki [Text] / D. Kazakov, V. Vol'hin, I. Zernina, D. Kosheleva // Vestnik Nizhegorodskogo universiteta im. N. I. Lobachevskogo. - 2008. Issue 3. - P. 69-72.

6. Yurchenko, V. Mathematical description of changes in specific rate of biological oxidation of methane [Text] / V. Yurchenko, A. Bahareva // Eastern-European Journal of Enterprise Technologies. - 2012. - Vol. 1, Issue 6 (55). - P. 4-6. - Available at: http:// journals.uran.ua/eejet/article/view/3388/3188

7. Ahmadullina, L. Matematicheskoe modelirovanie protsessa nizkotemperaturnoy parovoy konversii propana $\mathrm{v}$ prisutstvii metana na nikelevom katalizatore [Text] / L. Ahmadullina, L. Enikeeva, A. Novichkova, I. Gubaydullin // Zhurnal Srednevolzhskogo matematicheskogo obshchestva. - 2016. - Issue 3. - P. 117-126.

8. Yakovlev, I. Matematicheskoe modelirovanie protsessa polucheniya sintez - gaza v reaktore fil'tratsionnogo goreniya pri povyshennyh davleniyah [Text] / I. Yakovlev, S. Zambalov, V. Skripnyak // Vestnik Tomskogo Gosudarstvennogo Universiteta. 2014. - Issue 6. - P. 103-120.

9. Kuranov, A. Matematicheskoe modelirovanie konversii uglevodorodnogo topliva v elementah teplozashchity giperzvukovyh letatel'nyh apparatov [Text] / A. Kuranov, A. Korabel'nikov, A. Mihaylov // Zhurnal tekhnicheskoy fiziki. - 2017. - Vol. 87, Issue 1. - P. 27-33. doi: 10.21883/jtf.2017.01.44014.1856

10. Dehimi, L. Microkinetic modelling of methane dry reforming over Ni/Al2O3 catalyst [Text] / L. Dehimi, Y. Benguerba, M. Virginie, H. Hijazi // International Journal of Hydrogen Energy. - 2017. - Vol. 42, Issue 30. - P. 18930-18940. doi: 10.1016/ j.ijhydene.2017.05.231

11. Cruz, B. M. A two-dimensional mathematical model for the catalytic steam reforming of methane in both conventional fixed-bed and fixed-bed membrane reactors for the Production of hydrogen [Text] / B. M. Cruz, J. D. da Silva // International Journal of Hydrogen Energy. - 2017. - Vol. 42, Issue 37. - P. 23670-23690. doi: 10.1016/j.ijhydene.2017.03.019

12. Ghahraloud, H. Modeling and optimization of methanol oxidation over metal oxide catalyst in an industrial fixed bed reactor [Text] / H. Ghahraloud, M. Farsi // Journal of the Taiwan Institute of Chemical Engineers. - 2017. - Vol. 81. - P. 95-103. doi: 10.1016/j.jtice.2017.10.003

13. Vatani, A. Kinetic modeling of oxidative coupling of methane over $\mathrm{Li} / \mathrm{MgO}$ catalyst by genetic algorithm [Text] / A. Vatani, E. Jabbari, M. Askarieh, M. A. Torangi // Journal of Natural Gas Science and Engineering. - 2014. - Vol. 20. - P. 347-356. doi: 10.1016/j.jngse.2014.07.005

14. Popovich, A. Research into methane oxidation on oxide catalyst of the applied type [Text] / A. Popovich, G. Soloviev, A. Suvorin // Eastern-European Journal of Enterprise Technologies. - 2017. - Vol. 4, Issue 6 (88). - P. 29-34. doi: 10.15587/17294061.2017.107249 